\title{
Aliovalent titanium substitution in layered mixed Li Ni-Mn-Co oxides for lithium battery applications
}

\author{
Kinson C. Kam*a and Marca M. Doeff $* a$ \\ Received (in $X X X, X X X) X t h X X X X X X X X X 200 X$, Accepted $X t h X X X X X X X X X 200 X$ \\ ${ }_{5}$ First published on the web $X$ th $X X X X X X X X X 200 X$ \\ DOI: 10.1039/b000000x
}

Improved electrochemical characteristics are observed for $\mathrm{Li}\left[\mathrm{Ni}_{1 / 3} \mathrm{Co}_{1 / 3-\mathrm{y}} \mathrm{M}_{\mathrm{y}} \mathrm{Mn}_{1 / 3}\right] \mathrm{O}_{2}$ cathode materials when $\mathrm{M}=\mathrm{Ti}$ and $y<0.07$, compared to the baseline material, with up to $15 \%$ 10 increased discharge capacity.

Layered mixed transition metal oxides $\mathrm{Li}\left[\mathrm{Ni}_{\mathrm{x}} \mathrm{Co}_{1}\right.$. $\left.{ }_{2 \mathrm{x}} \mathrm{Mn}_{\mathrm{x}}\right] \mathrm{O}_{2}$, or NMCs, are attractive candidates for use as cathodes in lithium ion batteries due to their high capacity and the reduced Co content compared to $\mathrm{LiCoO}_{2}$. $\mathrm{Li}\left[\mathrm{Ni}_{1 / 3} \mathrm{Co}_{1 / 3} \mathrm{Mn}_{1 / 3}\right] \mathrm{O}_{2}$ delivers $15 \mathrm{up}$ to $160 \mathrm{mAh} / \mathrm{g}$ after charging to $4.2 \mathrm{~V}$ and $200 \mathrm{mAh} / \mathrm{g}$ to $\sim 4.7$ $\mathrm{V}$ in a lithium half cell configuration, and has better thermal abuse tolerance than $\mathrm{LiNi}_{0.8} \mathrm{Co}_{0.15} \mathrm{Al}_{0.05} \mathrm{O}_{2} .{ }^{1}$ The electrochemical activity is attributable to redox processes of $\mathrm{Ni}$ and $\mathrm{Co}$, whereas $\mathrm{Mn}$ remains in the +4 oxidation state during normal charge and 20 discharge. ${ }^{2}$ The cobalt in the transition metal layers of the NMCs decreases the number of $\mathrm{Li} / \mathrm{Ni}$ anti-site defects compared to the parent $\mathrm{LiNi}_{0.5} \mathrm{Mn}_{0.5} \mathrm{O}_{2}$, resulting in better rate performance. ${ }^{3}$ For vehicular applications, it is desirable, however, to reduce the cobalt content further, for reasons of cost, ideally without 25 compromising performance.

Towards this end, we have been investigating full or partial substitution of $\mathrm{Co}$ in NMCs with $\mathrm{Al}, \mathrm{Fe}$, or Ti. ${ }^{4}$ Our results showed that small amounts of $\mathrm{Al}$ or $\mathrm{Ti}$ are beneficial to electrochemical performance. However, aliovalent substitution of ${ }_{30} \mathrm{Co}$ (e.g. with Ti) is more complex than the corresponding isovalent substitution processes, due to the need for charge compensation. Several mechanisms for this are possible; for example, some $\mathrm{Mn}^{4+}$ may be replaced with $\mathrm{Mn}^{3+}$, or a lithium deficiency on $3 a$ sites may occur. It is also possible to substitute 2 ${ }_{35} \mathrm{Li}^{+}$ions with every $\mathrm{Ti}^{4+}$ ion on $3 b$ (transition metal) sites; this is effectively what occurs with the recently reported $x \mathrm{xiMn}_{0.5} \mathrm{Ni}_{0.5} \mathrm{O}_{2} \cdot 1-\mathrm{xLi}_{2} \mathrm{TiO}_{3}$ composites. ${ }^{5}$ To gain further insight on $\mathrm{Ti}$ substitution processes in NMCs, we have selected the $\mathrm{Li}\left[\mathrm{Ni}_{1 / 3} \mathrm{Co}_{1 / 3-\mathrm{y}} \mathrm{Ti}_{\mathrm{y}} \mathrm{Mn}_{1 / 3}\right] \mathrm{O}_{2}$ system and describe it in this 40 communication.

Details of the synthesis, electrode preparation, and experimental procedures are given in the electronic supplementary information. Table 1 lists the actual compositions of the materials used in this study, obtained by ICP analysis.

${ }_{45}$ Several samples contained a significant excess of lithium and their code names contain the symbol Li to designate this fact.

Table 1 Sample compositions of Ti-substituted NMCs

\begin{tabular}{cc}
\hline Composition & Code Name \\
\hline $\mathrm{Li}\left[\mathrm{Ni}_{0.33} \mathrm{Co}_{0.33} \mathrm{Mn}_{0.34}\right] \mathrm{O}_{2}$ & $\mathrm{Ti}-00$ \\
$\mathrm{Li}_{1.03}\left[\mathrm{Ni}_{0.34} \mathrm{Co}_{0.29} \mathrm{Ti}_{0.02} \mathrm{Mn}_{0.35}\right] \mathrm{O}_{2}$ & $\mathrm{Ti}-02$ \\
$\mathrm{Li}_{1.03}\left[\mathrm{Ni}_{0.35} \mathrm{Co}_{0.25} \mathrm{Ti}_{0.03} \mathrm{Mn}_{0.36}\right] \mathrm{O}_{2}$ & $\mathrm{Ti}-03$ \\
$\mathrm{Li}_{1.10}\left[\mathrm{Ni}_{0.38} \mathrm{Co}_{0.21} \mathrm{Ti}_{0.03} \mathrm{Mn}_{0.38}\right] \mathrm{O}_{2}$ & $\mathrm{Ti}-03-\mathrm{Li}$ \\
$\mathrm{Li}_{1.12}\left[\mathrm{Ni}_{0.38} \mathrm{Co}_{0.16} \mathrm{Ti}_{0.07} \mathrm{Mn}_{0.39}\right] \mathrm{O}_{2}$ & $\mathrm{Ti}-07-\mathrm{Li}$ \\
\hline
\end{tabular}

50
The powder diffraction patterns of the Ti-substituted NMCs are shown in Fig. 1, along with that of the parent compound. With the exception of sample Ti-07-Li, all peaks in all the patterns could be indexed to the $\mathrm{R} \overline{3} \mathrm{~m}$ space group. For ${ }_{55} \mathrm{Ti}-07-\mathrm{Li}$, an additional shoulder is observed at $2 \theta \approx 43.8^{\circ}$ suggesting the presence of a secondary phase. This is tentatively assigned to a spinel of unknown composition and suggests that the solubility limit for $\mathrm{Ti}$ is below about 7 atom percent. The substitution causes systematic shifts in peak positions and peak 60 broadening, indicating that $\mathrm{Ti}$ is incorporated in the transition metal layers. Preliminary Rietveld refinements (not shown) indicate that the unit cell volumes increase as Ti content goes up, as would be expected for substitution of the $\mathrm{Co}^{3+}$ ion with the larger $\mathrm{Ti}^{4+}$ ion. Scanning electron micrographs (SEMs) of these ${ }_{65}$ samples show that they consist of primary particles $\sim 75-100 \mathrm{~nm}$ in diameter and are highly agglomerated into larger secondary particles with micron-sized pores. Substitution does not affect the particle morphologies. Fig. 2 shows a typical SEM image of one of the samples.
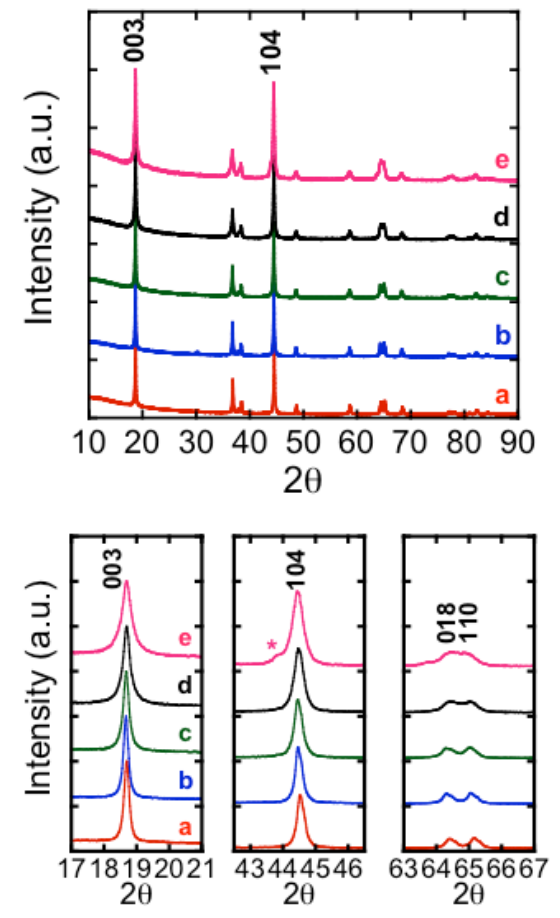

Fig. 1 Top: XRD powder patterns of (a) Ti-00, (b) Ti-02, (c) Ti03, (d) Ti-03-Li, and (e) Ti-07-Li. Bottom: Expansions of selected regions of the patterns. An asterisk marks an impurity peak at about $2 \theta \approx 44^{\circ}$ (middle). 


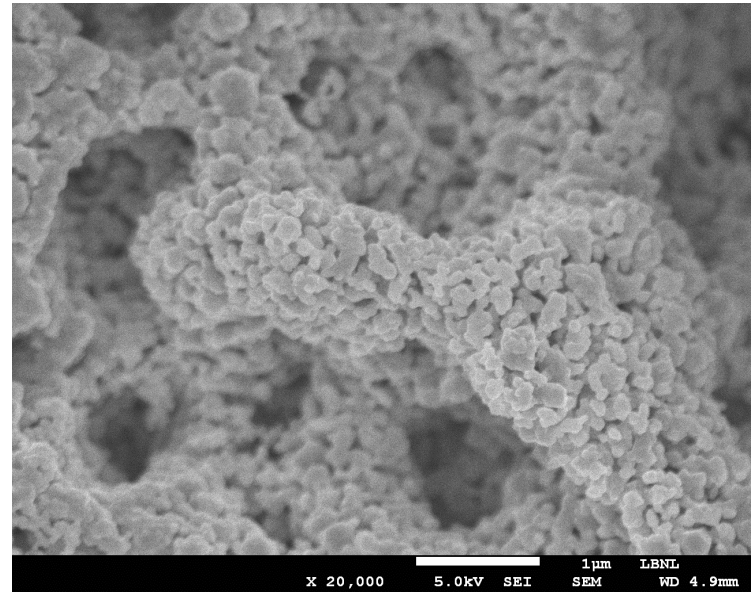

Fig. 2 SEM image of the Ti-00 powder. The morphology is typical of the NMCs used in this study.

Lithium half-cells containing the subject materials were cycled at $0.1 \mathrm{~mA} / \mathrm{cm}^{2}$ or about $\mathrm{C} / 10$ rate (Fig. 3). The discharge profiles after charge to $4.3 \mathrm{~V}$ are typical of stoichiometric NMCs, but samples Ti-02, Ti-03 and Ti-03-Li deliver somewhat higher 10 specific capacities than Ti-00. In contrast, Ti-07-Li is markedly inferior, most likely due to the presence of the impurity phase. When the charge limit is extended to $4.7 \mathrm{~V}$, the differences between the Ti-substituted compounds and the baseline material are even more apparent. The specific capacity is increased by as s much as $15 \%$ (up to $235 \mathrm{mAh} / \mathrm{g}$ ) for several of the Ti-substituted samples. Furthermore, the two compounds with the greatest lithium excess, Ti-03-Li and Ti-07-Li, show an additional feature in the discharge profile at about $3.0 \mathrm{~V}$, which is not evident in profiles after charging to $4.3 \mathrm{~V}$. This is suggestive of an 20 activation process whereby electro-active $\mathrm{Mn}$ is generated, similar to that which occurs in Li-excess NMCs and composite materials (e.g., those containing a $\mathrm{Li}_{2} \mathrm{MnO}_{3}$ component) after charge to high potentials; i.e. concomitant loss of lithium and oxygen and structural rearrangement. ${ }^{6}$ If so, this indicates that 25 some of the excess lithium is indeed present in the transition metal layers ( $3 b$ sites), rather than in an impurity phase on particle surfaces (e.g., $\mathrm{Li}_{2} \mathrm{CO}_{3}$ ). Ti-02 and Ti-03, in contrast, do not show this feature, do not contain a large excess of lithium, and deliver nearly the same very high capacity as Ti-03-Li. Thus, 30 neither the presence of excess lithium on $3 b$ sites nor an activation process is essential for delivering the higher capacities observed for some of the Ti-substituted NMCs. Furthermore, these observations suggest that charge compensation can occur either by co-substitution of $\mathrm{Li}$ on $3 b$ sites or by one of the other 35 mechanisms suggested above.

The origins of the increased capacities for several of the Ti-substituted samples during $0.1 \mathrm{~mA} / \mathrm{cm}^{2}$ discharges, are, as yet, not completely understood. The rate capabilities are not substantially different than that of the baseline NMC, but higher

40 capacities are obtained at every current density tested between 0.1 and $2.0 \mathrm{~mA} / \mathrm{cm}^{2}$ (not shown) for samples not containing excess lithium. To minimize kinetic effects, lithium half-cells containing the subject cathodes were potentiostatically stepped between 4.3 and $2.0 \mathrm{~V}$ in $10 \mathrm{mV}$ increments at a rate equivalent to about $\mathrm{C} / 50$.

${ }_{45}$ Somewhat higher specific capacities were obtained in all cases, compared to the galvanostatic discharges at $0.1 \mathrm{~mA} / \mathrm{cm}^{2}$. Table 2 includes the results of these experiments, including the observed first cycle irreversible capacities expressed as a percentage of the initial charge capacity.
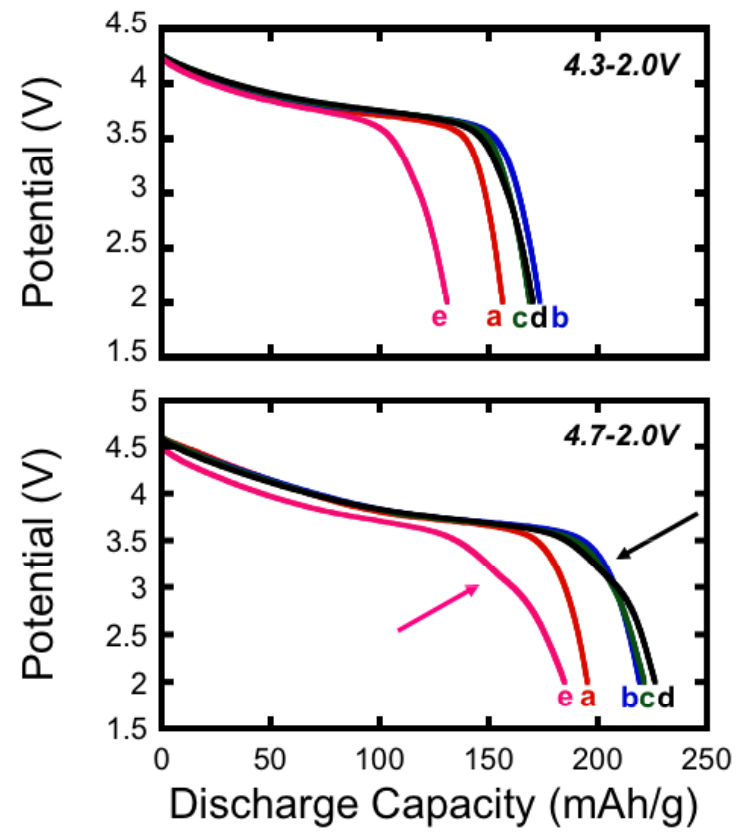

Fig. 3 Discharges at $0.1 \mathrm{~mA} / \mathrm{cm}^{2}$ from $4.3-2.0 \mathrm{~V}$ (top) or $4.7-2.0 \mathrm{~V}$ (bottom) of lithium cells containing (a) Ti-00, (b) Ti-02, (c) Ti03, (d) Ti-03-Li, and (e) Ti-07-Li. Arrows point to the additional feature at $3.0 \mathrm{~V}$ in the discharge profiles from $4.7-2.0 \mathrm{~V}$ of cells ${ }_{55}$ containing Ti-03-Li and Ti-07-Li.

Table 2 Results of slow potentiostatic experiments on lithium half cells containing Ti-substituted NMCs

\begin{tabular}{ccc}
\hline Sample & Disch. Cap., mAh/g & \% Irrev. Cap. \\
\hline Ti-00 & 184 & 7.3 \\
Ti-02 & 196 & 5.8 \\
Ti-03 & 190 & 5.4 \\
Ti-03-Li & 182 & 8.7 \\
Ti-07-Li & 182 & 12 \\
\hline
\end{tabular}

60

Relatively high first cycle coulombic inefficiencies are characteristic of cells containing NMC materials and are observed even after partial charging. ${ }^{7}$ It has been suggested that lithium diffusion becomes increasingly sluggish as the $3 a$ sites become ${ }_{65}$ nearly fully occupied, making it difficult to recover all of the charge capacity under normal conditions. The observation that the so-called irreversible capacity can be completely recovered after discharge to low voltages supports the hypothesis that kinetic limitations are responsible for the phenomenon. The 70 results in Table 2 show that first cycle irreversible capacities are somewhat lower in cells containing Ti-02 and Ti-03 than in the one with Ti-00. Cells with Ti-03-Li and Ti-07-Li, however, have comparatively high irreversible capacities, perhaps due to the onset of the activation processes discussed above. It is possible to 75 explain the lower irreversible capacities in the Ti-02 and Ti-03 electrodes if lithium deficiencies in the $3 a$ sites compensate for the aliovalent substitution. This would lower the apparent coulombic inefficiencies, by reducing the initial charge capacities. However, the discharge capacities should remain the 80 same as in the parent compound (or decrease as a consequence of a higher degree of ion-mixing, as often happens with materials synthesized with lithium deficiencies). The increased discharge capacities suggest that charge compensation in the substituted 
NMCs occurs via replacement of a small amount of $\mathrm{Mn}^{4+}$ with $\mathrm{Mn}^{3+}$ instead. The mixed oxidation states for Mn may result in higher electronic conductivities for the fully lithiated $\mathrm{Ti}$ substituted compounds. If electronic rather than ionic 5 conductivity limitations cause the observed kinetic sluggishness near the end of discharge in cells containing NMCs, this may explain the positive effects of Ti substitution that are observed. This decreases the apparent irreversibility, resulting in the higher discharge capacities that are seen. This, however, requires further 10 study.

Better capacity retention upon cycling is observed for cells containing several of the Ti-substituted materials, regardless of the voltage window used (Fig. 4). Although some NMCs can deliver high capacities after charging to high potentials, fading is 15 often severe (as is seen for Ti-00 in this example). Composite $\left(\mathrm{Li}_{2} \mathrm{MnO}_{3}\right.$ stabilized) materials with high capacities usually exhibit better cycling behavior, but require the activation process discussed earlier. The loss of oxygen damages particle surfaces, resulting in poor rate capability. This initial result for the Ti20 substituted materials suggest that it may be possible to obtain a stable higher capacity upon cycling than is usual for the stoichiometric NMCs, without needing activation or sacrificing rate capability.

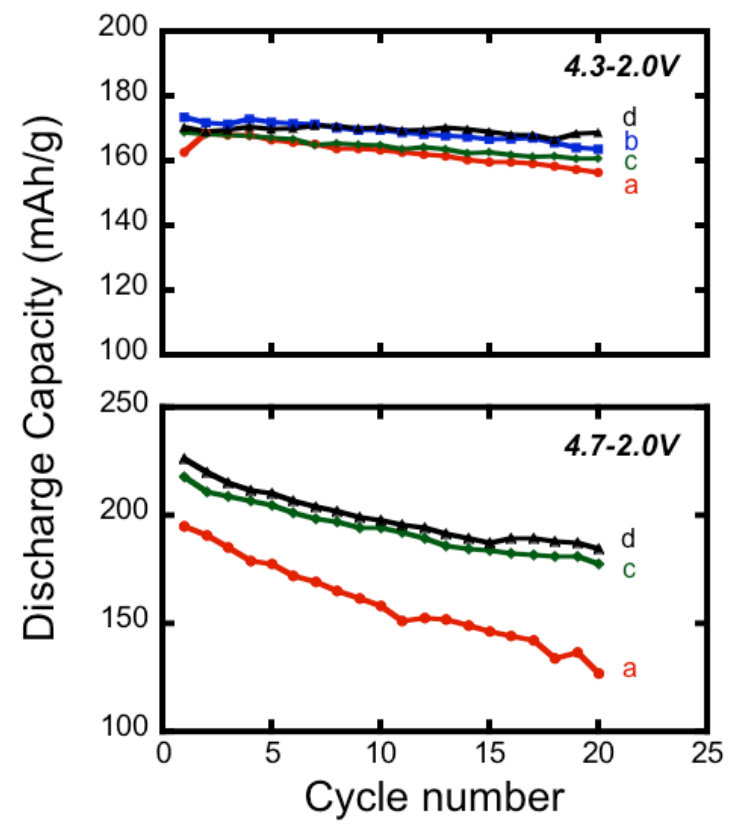

${ }_{25}$ Fig. 4 Discharge capacity as a function of cycle number for lithium cells containing (a) Ti-00, (b) Ti-02, (c) Ti-03, and (d) Ti03-Li. Cells were discharged at $0.1 \mathrm{~mA} / \mathrm{cm}^{2}$ between 4.3 or 4.7 and $2.0 \mathrm{~V}$.

In summary, partial substitution of $\mathrm{Ti}$ for $\mathrm{Co}$ in $30 \mathrm{Li}\left[\mathrm{Ni}_{1 / 3} \mathrm{Co}_{1 / 3-\mathrm{y}} \mathrm{M}_{\mathrm{y}} \mathrm{Mn}_{1 / 3}\right] \mathrm{O}_{2}$ cathodes results in higher discharge capacities and better cycling characteristics in cells containing them. The mechanism of the aliovalent substitution is of interest and influences the observed electrochemical properties. In some cases, $\mathrm{Li}$ co-substitutes with $\mathrm{Ti}$ on transition metal sites to 35 compensate for charge, resulting in a lithium-excess material. The higher discharge capacity after charge to $4.7 \mathrm{~V}$ is probably attributable to an activation process similar to that seen in composite $\mathrm{NMC}$ materials containing a $\mathrm{Li}_{2} \mathrm{MnO}_{3}$ component, in which both $\mathrm{Li}$ and oxygen are removed at high potentials. In
40 other cases, the charge compensation occurs, most likely, by replacement of some $\mathrm{Mn}^{4+}$ with $\mathrm{Mn}^{3+}$, and the higher capacity that is observed is not contingent upon an activation process. The reasons for the improvement are not completely understood, but may be the result of higher conductivities in the as-made 45 materials that arise as a result of mixed valence states for $\mathrm{Mn}$. The higher capacities do not come at the expense of poorer rate or cycling behavior, suggesting that low levels of aliovalent $\mathrm{Ti}$ substitution may be a route to preparing NMC materials with superior electrochemical properties, without the disadvantages of ${ }_{50}$ composite materials. Further work on this system is planned in order to investigate the origins of the improvement and will be reported in a subsequent publication.

\section{${ }_{55}$ Acknowledgments}

This work was supported by the Assistant Secretary of Energy for Energy Efficiency and Renewable Energy, Office of Vehicle Technologies of the United States Department of Energy under Contract No. DE-AC02-05CH11231. 60

\section{Notes and references}

${ }^{a}$ Lawrence Berkeley National Laboratory, Environmental Energy Technologies Division, University of California, Berkeley, CA. 94720. U.S.A. Fax: 510486 4881; Tel: 510486 5821;E-mail: mmdoeff@lbl.gov $65 \dagger$ Electronic Supplementary Information (ESI) available: [Methods and Characterization, and Electrochemical Cell Assemblies]. See DOI: $10.1039 / \mathrm{b} 000000 \mathrm{x}$

1. I. Belharouak, W. Q. Lu, D. Vissers and K. Amine, Electrochem. 70 Commun., 2006, 8, 329

2. (a) N. Yabuuchi, Y. Koyama, N. Nakayama and T. Ohzuku, $J$ Electrochem. Soc., 2005, 152, A1434; (b) T. Ohzuku and Y. Makimura, Chem. Lett., 2001, 30, 642; (c) N. Yabuuchi and T. Ozhuku, J. Power Sources, 2003, 119-121, 171; (d) Y. Koyama, N.

75 Yabuuchi, I. Tanaka, H. Adachi and T. Ohzuku, J. Electrochem Soc., 2004, 151, A1545; (e) N. Yabuuchi, Y. Makimura and T. Ohzuku, J. Electrochem. Soc., 2007, 154, A314.

3. (a) A. Van der Ven, G. Ceder, M. Asta and P. D. Tepesch, Phys. Rev. B, 2001, 64, 184307; (b) A. Van der Ven and G. Ceder, $J$ Power Sources, 2001, 97-98, 529; (c) K. Kang and G. Ceder, Phys. Rev. B, 2006, 74, 094105; (d) A. Rougier, P. Saadoune, P. Gravereau, P. Willmann and C. Delmas, Solid State Ionics, 1996, $143,1168$.

4. (a) J. Wilcox, S. Patoux and M. Doeff, J. Electrochem. Soc., 2009, 85 156, A192; (b) J. D. Wilcox, E. E. Rodriguez and M. M. Doeff, $J$ Electrochem. Soc., 2009, 156, A1011.

5. (a) C. S. Johnson, J. S. Kim, A. J. Kropf, A. J. Kahaian, J. T. Vaughey and M. M. Thackeray, Electrochem. Commun., 2002, 4, 492. (b) J. S. Kim, C. S. Johnson and M. M. Thackeray, Electrochem. Commun., 2002, 4, 205.

6. M. M. Thackeray S. H. Kang, C. S. Johnson, J. T. Vaughey, R. Benedek, and S. A. Hackney, J. Mater. Chem., 2002, 17, 3112.

7. S. H. Kang, W. S. Yoon, K. W. Nam, X. Q. Yang, and D. P. Abraham, J. Mater. Sci., 2008, 43, 4701. 